\title{
Coronal partings
}

\author{
Igor F. Nikulin ${ }^{\mathrm{a}, *}$, Yurii V. Dumin ${ }^{\mathrm{a}, \mathrm{b}, \mathrm{c}}$ \\ ${ }^{a}$ P.K. Sternberg Astronomical Institute of M.V. Lomonosov Moscow State University, \\ Universitetskii prosp. 13, 119234, Moscow, Russia \\ ${ }^{b}$ Space Research Institute of Russian Academy of Sciences, \\ Profsoyuznaya str. 84/32, 117997, Moscow, Russia \\ ${ }^{c}$ Max Planck Institute for the Physics of Complex Systems, \\ Noethnitzer Str. 38, 01187, Dresden, Germany
}

\begin{abstract}
The basic observational properties of "coronal partings" - the special type of quasi-one-dimensional magnetic structures, identified by a comparison of the coronal X-ray and EUV images with solar magnetograms - are investigated. They represent the channels of opposite polarity inside the unipolar large-scale magnetic fields, formed by the rows of magnetic arcs directed to the neighboring sources of the background polarity. The most important characteristics of the partings are discussed. It can be naturally assumed that - from the evolutionary and spatial points of view - the partings can transform into the coronal holes and visa versa. The classes of global, intersecting, and complex partings are identified.
\end{abstract}

Keywords: solar magnetic fields, coronal structures

\section{Introduction}

X-ray investigations of the Sun in the recent decades revealed a number of specific coronal structures, such as the bright X-ray points (Golub et al., 1974) and coronal holes (Altschuler et al., 1972; Fuerst \& Hirth, 1975; Timothy et al., 1975; Nolte et al., 1976). The coronal holes (CH) attracted especial attention after discovery of their geoefficiency (Krieger et al., 1973; Neupert \& Pizzo, 1974, Nolte et al., 1976). However, genetic and spatial relations

\footnotetext{
${ }^{*}$ Corresponding author

Email addresses: ifn@sai.msu.ru (Igor F. Nikulin), dumin@yahoo.com (Yurii V. Dumin)
} 
between $\mathrm{CH}$ and other coronal structures and magnetic fields are still investigated poorly. So, it is interesting to look for the additional large-scale morphological features in the solar corona and to reveal their relation to the previously-known ones.

With this aim in view, during the last 15 years we performed a visual inspection of a large number of images regularly provided by the leading spaceand ground-based observatories. First of all, we used the data by Yohkoh SXT $^{1}$ (Tsuneta et al., 1991) and SOHO EIT (Delaboudinière et al., 1995). In the recent time, we began to employ also the very-high-quality images by SDO AIA (Pesnell et al., 2012). To confront the coronal structures visible in soft X-rays and extreme ultraviolet (EUV) with the associated magnetic fields, we used the observations of photospheric magnetic fields mostly by SOHO MDI ${ }^{2}$ (Scherrer et al. , 1995) and, recently, by SDO HMI (Pesnell et al., 2012). Synoptic magnetic maps by the ground-based observatories were also occasionally employed. In total, we have analyzed over 4000 spectroheliograms and magnetograms, starting from 1999 ? $^{3}$

In particular, we searched for the characteristic elongated X-ray and ultraviolet structures with a reduced brightness, which were associated with the unipolar regions. (It is necessary to discriminate them from the filaments, which are localized at the boundaries between different polarities.) As a result, it was found that the magnetic field lines, represented by the X-ray loops, over the regions of magnetic field of the same sign often form the specific channels between two rows of the arcs directed oppositely, i.e. toward the regions of another polarity (Fig. 1). Because of their similarity to a hair parting - where hairs play the role of the field lines - Nikulin (2003) suggested to call them the coronal partings (CP).

A few years later, Molodenskii \& Starkova (2007) justified theoretically formation of such partings and performed their computer simulation. They also concluded that these partings are the inevitable and natural component of the magnetized solar atmosphere, representing the "sign-reversal lines for

${ }^{1}$ The most part of original data utilized in the present paper were downloaded from the archive at http://www.lmsal.com/SXT, which was later relocated to another address: http://ylstone.physics.montana.edu/ylegacy/.

${ }^{2}$ The original data are available in the archive: http://sohowww.nascom.nasa.gov.

${ }^{3}$ Unfortunately, the so long period of data collection resulted in some nonuniformity of the graphic formats as well as a number of parasitic designations in the images presented below. 
Partings of background fields

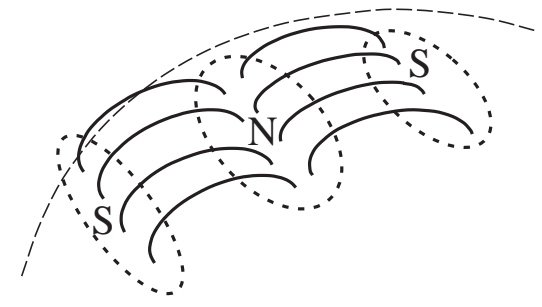

Yohkoh/SXT 2000-08-16 08:44
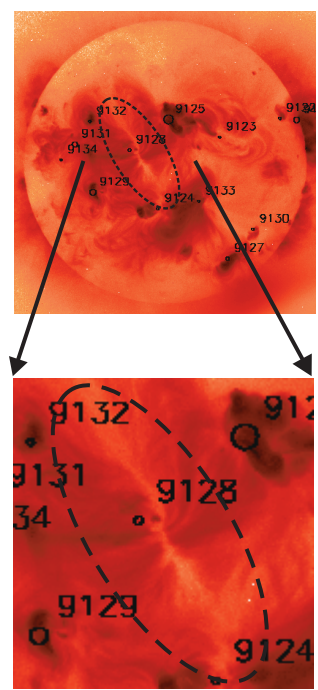

$\mathrm{SOHO} / \mathrm{MDI}$

2000-08-16 17:36

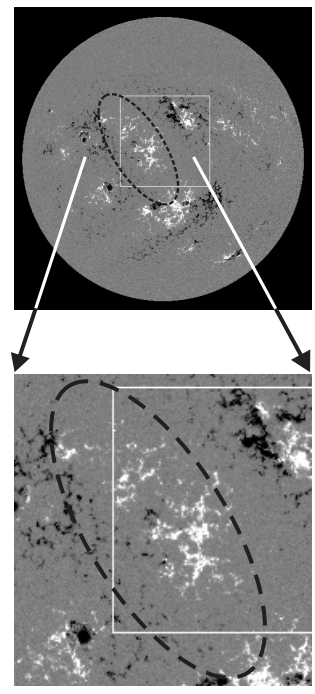

Partings of active regions

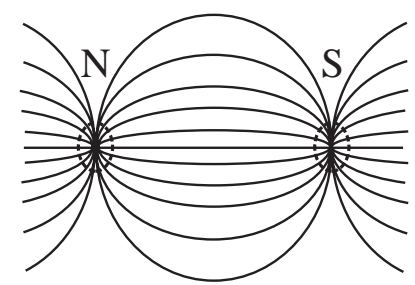

\section{Yohkoh/SXT} 2001-02-08 12:46

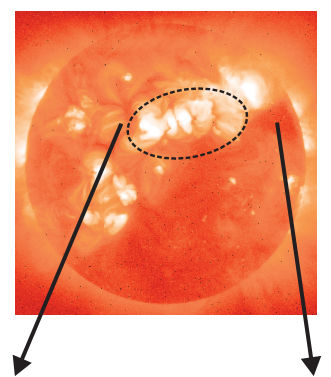

$\mathrm{SOHO} / \mathrm{MDI}$

2001-02-08 17:41

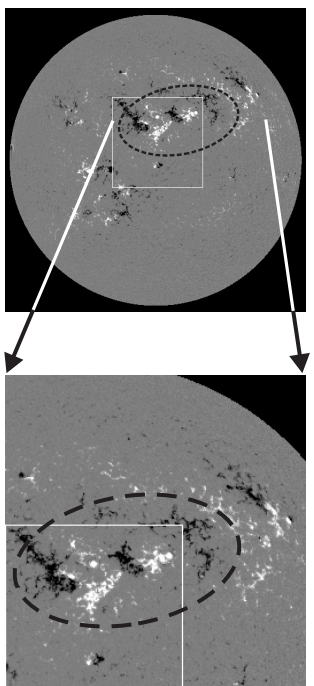

Figure 1: Scheme of the partings of background fields with an example for 16 August 2000 (left-hand side) and of the active regions with an example for 08 February 2001 (right-hand side). The patterns of soft X-ray emission (left column) and photospheric magnetic field (right column) are given for each case. The images by Yohkoh SXT and $S O H O$ MDI involve a number of irrelevant designations, which should not be taken into account. Besides, Yohkoh SXT images in the left- and right-hand sides are in the inversed palettes (negative and positive). 
the normal component of the curvature vector of the magnetic lines".

So, it is the aim of the present paper to give a short review of the most important morphological features of $\mathrm{CP}$, revealed in the course of our long-term investigations, as well as to discuss their relation to other coronal structures.

\section{Basic morphological features}

\subsection{Definitions}

The coronal partings can be subdivided into two groups: CP of the largescale background fields (Fig. 1, left-hand side) and the partings of active regions (AR), which represent the short and narrow channels inside the strong fields of AR (Fig. 1, right-hand side). In such a case, the most fraction of the magnetic flux of AR and the parting is enclosed between the leading and trailing parts of the sunspot group. However, if there are other AR or floccules nearby, then the peripheral part of the flux is connected to them. As a result, a short narrow channel is formed between the deviating field lines. It is usually located perpendicularly to the axis of the group and passes through the sunspots themselves. When the sunspots disappear and the respective magnetic fields decay, the coronal partings of active regions can be transformed into $\mathrm{CP}$ of background fields.

\subsection{Distinction between the coronal partings and holes}

To emphasize that $\mathrm{CP}$ and $\mathrm{CH}$ are different structures, let us formulate the main differences between them:

1. The magnetic fields of $\mathrm{CH}$ are weak and, at the level of sensitivity of apparatus, are almost absent. On the other hand, according to $\mathrm{SOHO}$ MDI magnetograms, the magnetic field of a typical $\mathrm{CP}$ is about 10 $50 \mathrm{Gs}$ in the partings of background fields and a few hundred Gs in the $\mathrm{CP}$ of active regions.

2. The coronal holes, by definition, are the coldest structures of the corona (Timothy et al., 1975), while the coronal partings possess a higher temperature and brightness.

3. The coronal holes usually occupy large areas on the Sun and do not have the elongated shape, characteristic of the coronal partings. From the mathematical point of view, $\mathrm{CP}$ is associated with a quasi-onedimensional line; while $\mathrm{CH}$, with a two-dimensional object (oval). 
Yohkoh/SXT

2001-02-15 13:07
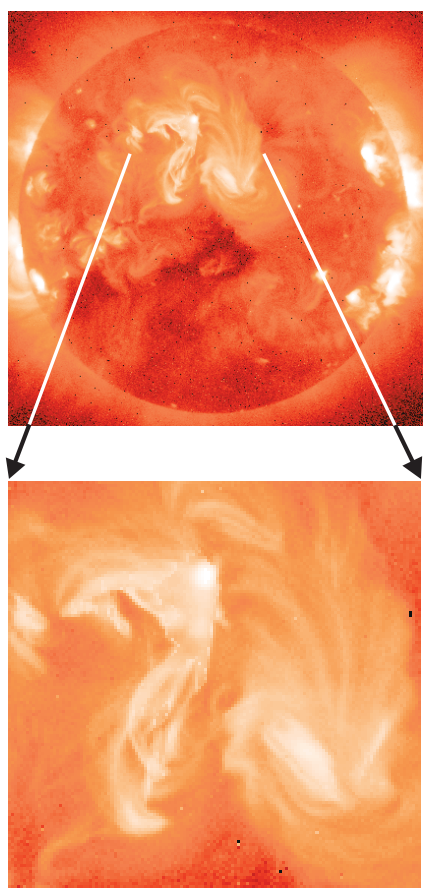

$\mathrm{SOHO} / \mathrm{MDI}$

2001-02-15 12:53
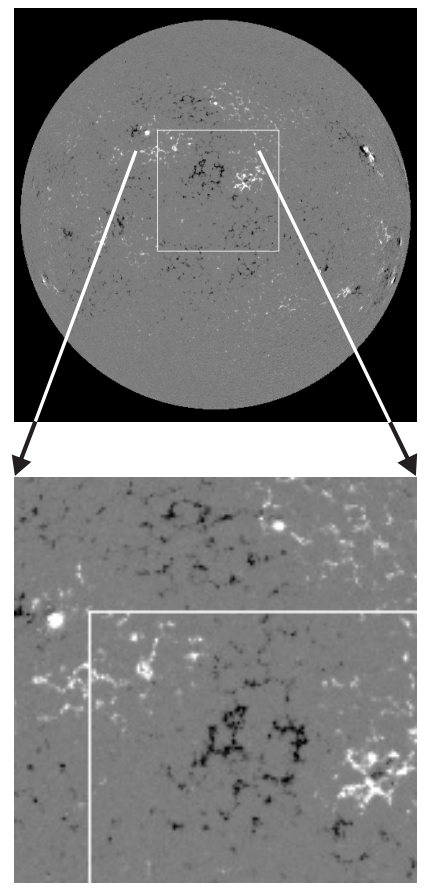

Yohkoh/SXT 2001-02-15 14:46
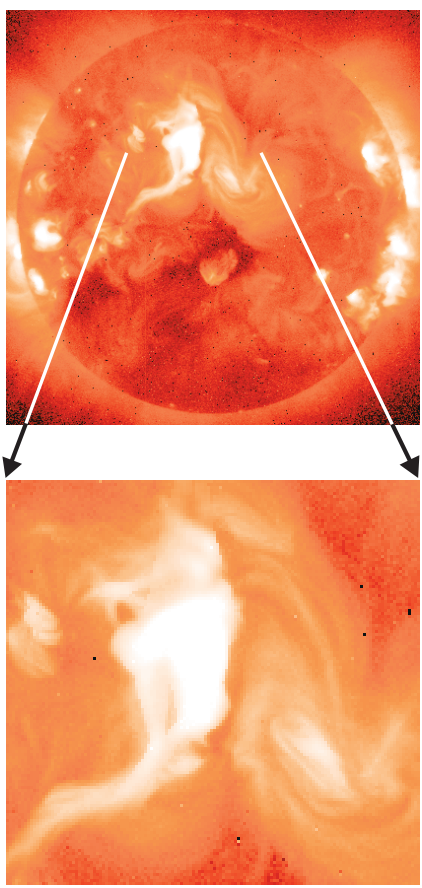

Figure 2: Asymmetric parting in the northern hemisphere on 15 February 2001: the patterns of soft X-ray emission (left and right columns) and photospheric magnetic field (central column).

4. The coronal holes are usually observed in the polar regions and less frequently in the low latitudes (Munro \& Withbroe, 1972), while the coronal partings usually emerge in the so-called "royal latitudes".

5. The coronal holes are observed more frequently near the minimum of solar activity; while the coronal partings are scarce near the minimum, most probably, because of the weakness (low structural contrast) of the corona and absence of the visible coronal loops, identifying CP.

Nevertheless, all these differences do not exclude the possibility of evolution of $\mathrm{CP}$ into $\mathrm{CH}$ in the course of decay of the X-ray structures and magnetic fields. Moreover, the above-listed features emphasize the similarity of these coronal structures, associated with presence of the open field lines. 


\subsection{Structure of the coronal partings}

The structure of $\mathrm{CP}$ and their lifetime are determined by the background (photospheric) magnetic fields and the ambient arc formations. Each knot in the magnetogram is a footpoint of the respective coronal arc with lifetime about a day (and sometimes longer). We believe that the evolution of $\mathrm{CP}$ is usually connected with the emergence of small bipolar regions - the bright X-ray points (BXRP) - forming their own system of arcs, associated with new ambient fields, and followed by the decrease in contrast and blurring the parting. If the CP-forming arcs weaken, then the parting expands, darkens, and can be transformed into $\mathrm{CH}$.

Roughly speaking, the X-ray brightness of $\mathrm{CP}$ is associated with the magnetic-field gradients: the stronger is variation of the field, the greater is the X-ray brightness of the corresponding structure. Namely, the brightness is higher in the case of $\mathrm{CP}$ interaction with a neighboring active region as compared to the case of the arcs directed to a weak floccule. As a result, the partings with substantially different brightness at the opposite sides (i.e., the asymmetric partings) can emerge. A typical example took place on 15 February 2001: a flare process developed in the northern hemisphere near the central meridian, while the asymmetric parting with an enhanced eastern part was seen slightly to the west (Fig. 2). It can be assumed from these images that the magnetic-field arcades of $\mathrm{CP}$ serve as a "barrier" for propagation of the flare.

As is seen in the soft X-ray images by Yohkoh, the active-region CP (Fig.1, right-hand side) usually passes through sunspots and separates the parts of the magnetic flux linked to the sunspots of opposite polarity in the corresponding group and to the peripheral magnetic fields. The same behavior can be observed in EUV images by $S D O$.

It is important that if $\mathrm{CP}$ intersects an equator, then sign of the respective magnetic field does not change, as distinct from AR, whose signs of the leading polarity are different in the opposite hemispheres.

\subsection{Magnetic-field topology in the partings}

Despite the fact that CP (for example, in the images of lines of the multiply-ionized iron, 171 and $195 \AA$ ) look like the channels of filaments, they are absolutely different structures from the viewpoint of magnetic topology. The filament channels and filaments themselves are formed at the boundary of the magnetic polarity (Martres et al., 1966), i.e. near the neutral line of the longitudinal magnetic field; while CP are formed in the regions of the same 
Yohkoh/SXT

1999-09-06 07:25

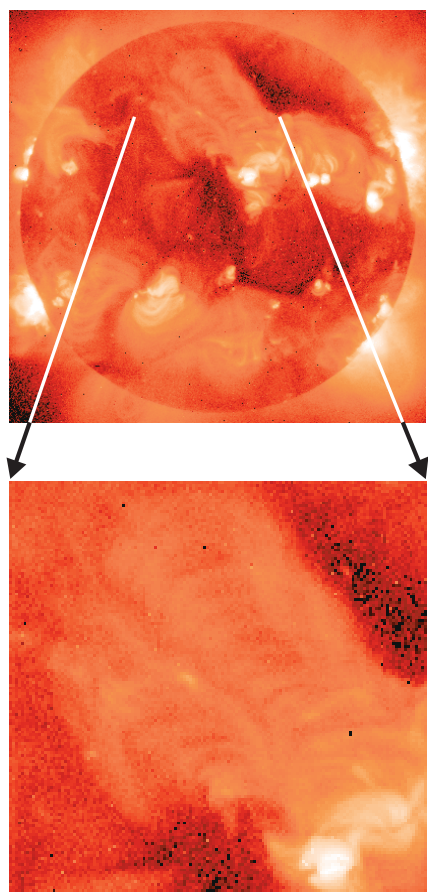

$\mathrm{SOHO} / \mathrm{MDI}$

1999-09-06 14:29
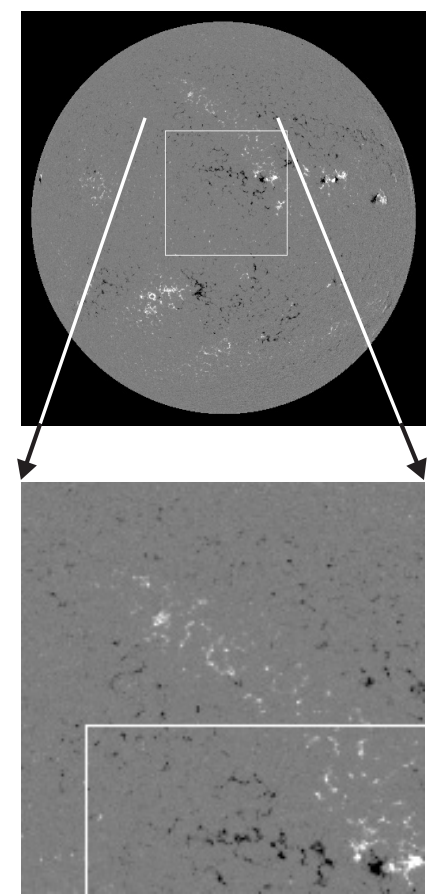

Kanzelhöhe Solar Obs., $\mathrm{H}_{\alpha}$ 1999-09-06 07:58

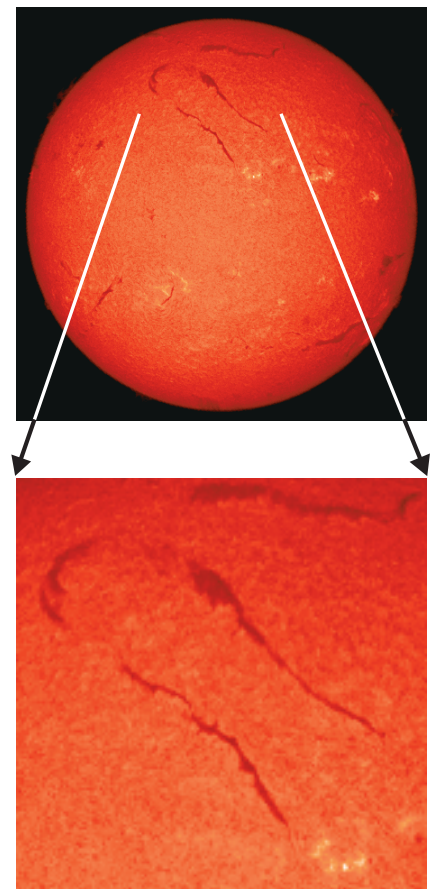

Figure 3: Coronal parting between two filaments on 06 September 1999: the patterns of soft X-ray emission (left column), photospheric magnetic field (central column), and $\mathrm{H}_{\alpha}$ emission (right column).

sign (Nikulin, 2006). In other words, CP emerge between the footpoints of arcs of the same polarity; while the filaments, at the top of these arcs, near the neutral line. In some cases, CP can be formed between two parallel filaments (e.g., the interesting case on 06 September 1999 in the northern hemisphere, presented in Fig. 3).

Since CP are localized at relatively low altitudes, they are often overlapped by the high-altitude X-ray structures. So, when from the analysis of magnetograms $\mathrm{CP}$ can be assumed to exist near the limb, they may be invisible in the X-ray images.

As regards the global magnetic topology, CP can be associated with a separatrix. In the simplest case of a narrow straight parting, it will represent a plane normal to the photosphere. Generally speaking, not every 
coronal parting is the separatrix (i.e., separates two globally-distinct magnetic fluxes): this should be proved by the magnetic field calculations in each particular case. And vice versa, not every separatrix is identical to the parting (e.g., Somov, 2013, Ch. 4): for example, if there is a sufficiently strong horizontal component of the magnetic field along the separatrix, then it will hardly manifest itself as CP. Therefore, the separatrices coincide with partings only in the particular cases; so that $\mathrm{CP}$ can be reasonably considered as the specific morphological structures in the solar corona.

\subsection{The coronal partings in different spectral lines}

The structure of CP in the infrared line of helium $10830 \AA$ is weak and smeared out: like $\mathrm{CH}$, they are only a few percent brighter than the background (as an example, see bottom left panel in Fig. 4).

In the $\mathrm{H}_{\alpha}$ line, the partings are identified by a weak emission, while in the magnetograms they often exhibit themselves as the parallel rows of unipolar magnetic knots - footpoints of the arcs forming the partings.

Apart from the X-ray images, CP are well distinguishable in EUV lines FeXV $284 \AA$ (e.g., right panel in Fig. 5 , below). On the other hand, in the lines Fe X and Fe XII they are almost indistinguishable from the channels of filaments.

\section{Types of the coronal partings and their evolution}

\subsection{Global coronal partings}

One can often observe the global structures with a decreased X-ray brightness, when a background or active-region CP passes from a polar coronal hole to the $\mathrm{CH}$ at another pole (for example, on 23-24 February 2001 and 01 August 2000, Fig. 4). The total length of these structures is, therefore, comparable to the solar diameter, while partings represent the links between $\mathrm{CH}$ localized in the opposite hemispheres. The lifetime of such CP is comparable to or somewhat less than the lifetime of corresponding $\mathrm{CH}$. The low-latitude part of $\mathrm{CP}$ is usually placed to the west with respect to the high-latitude part. This seems to be caused by a differential rotation during the long lifetime of these structures. Two examples of the global structures in the X-ray and infrared spectroheliograms and magnetograms are presented in Fig. 4. 

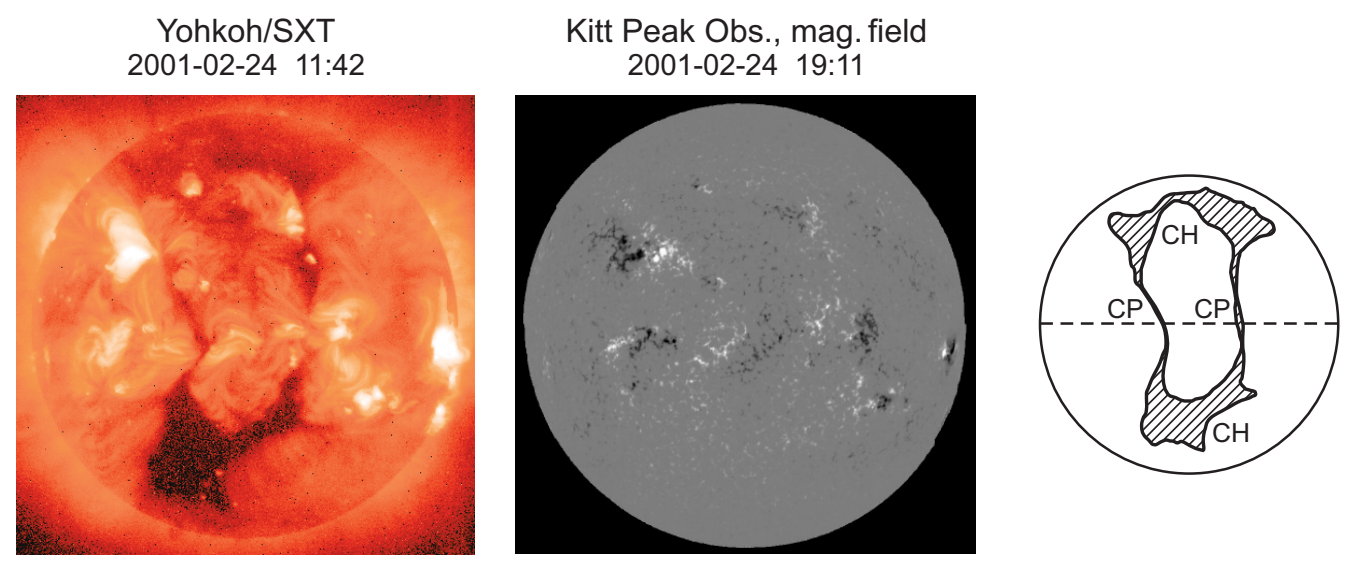

Yohkoh/SXT

2000-08-01 10:07

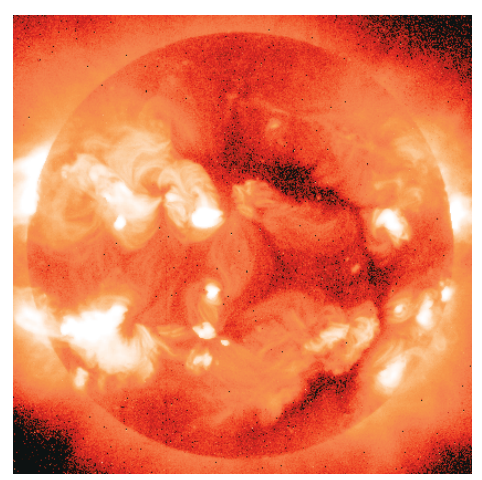

Kitt Peak Obs., Hel $10830 \AA$ 2000-07-30 16:59
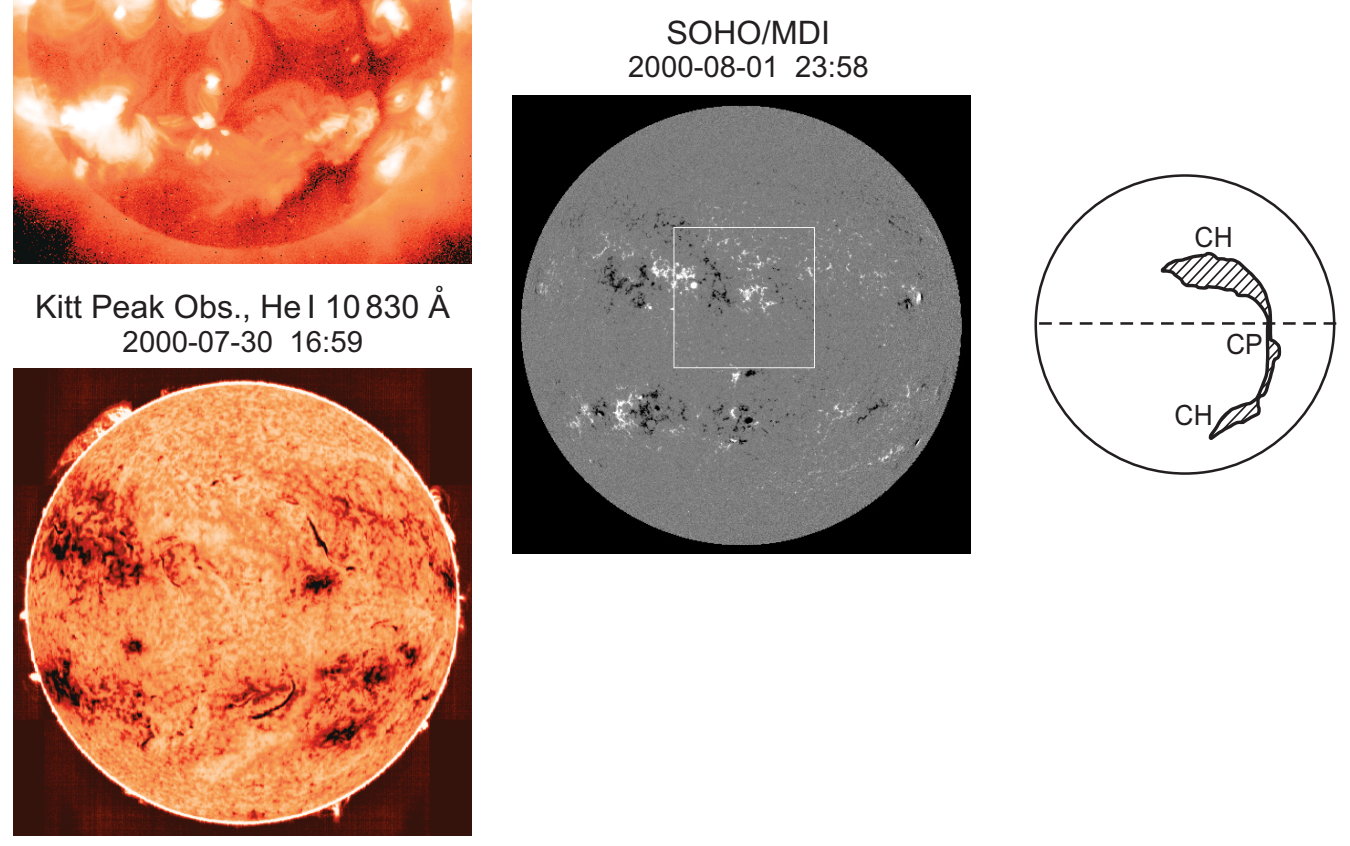

Figure 4: The global coronal partings on 23-24 February 2001 (top) and 01 August 2000 (bottom): the patterns of soft X-ray and infrared emission (left column, upper and lower panels respectively), photospheric magnetic field (central column), and schemes of the basic coronal structures (right column). 

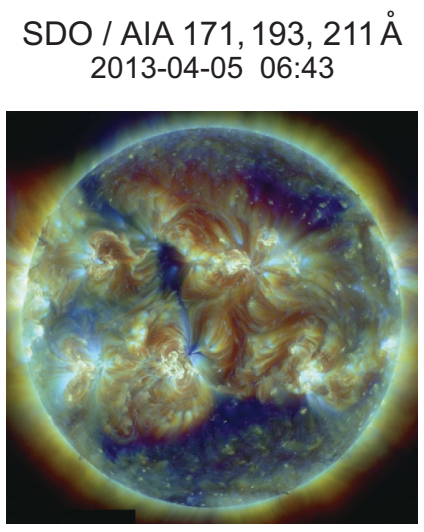
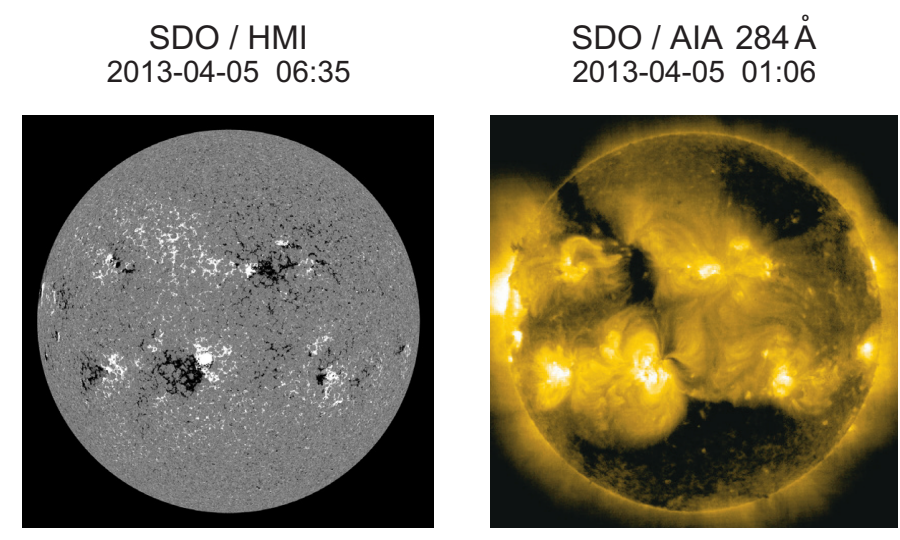

Figure 5: The complex coronal parting on 05 April 2013: patterns of EUV emission (left and right panels) and photospheric magnetic field (central panel) by SDO satellite.

\subsection{Complex coronal partings}

The complex (or composite) partings can be formed by both the activeregion and background CP. As an example, we can consider the data by $S D O$ satellite on 05 April 2013, presented in Fig. 5 a global parting passes from the north-east limb through the equator and a large sunspot in the south-east and finally terminates at $\mathrm{CH}$ in the southern polar region. This parting is best seen in Fe XV $284 \AA$ line. It is composed of a few different parts: (1) from the $\mathrm{NE}$ limb to a small $\mathrm{CH}$ near the center of the disc at $\sim 15 \mathrm{E} 25 \mathrm{~N}$, (2) through this $\mathrm{CH}$, after the equator, it passes between filaments of a large sunspot, (3) through the sunspot and between the filaments of superpenumbra in its southern part, up to the extensive $\mathrm{CH}$ in the south; the magnetic polarity of the photospheric background remaining always the same.

\subsection{Intersecting coronal partings}

As was already emphasized above, each $\mathrm{CP}$ is associated with a magnetic field of the same polarity. However, the partings with different polarities can intersect each other in the course of evolution and, as a result, the Xpoint should be formed (e.g., on 01 December 2000 and 01 March 2001, as illustrated in Fig. 6). As follows from the magnetograms, one of polarities dominates in the point of intersection. The role of such X-type partings in the physical processes, for example, in the solar flares is still to be clarified. 
Yohkoh/SXT

2001-03-01 03:50

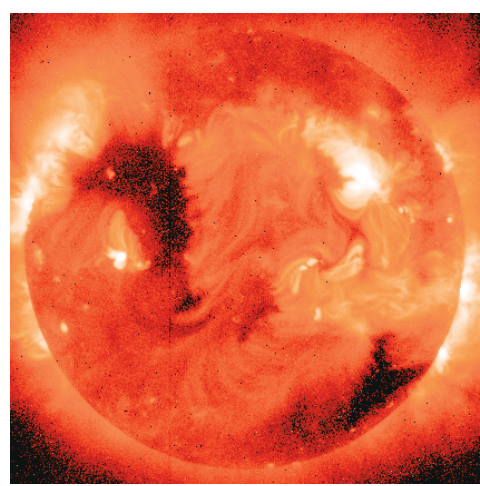

Yohkoh/SXT 2000-12-01 04:52

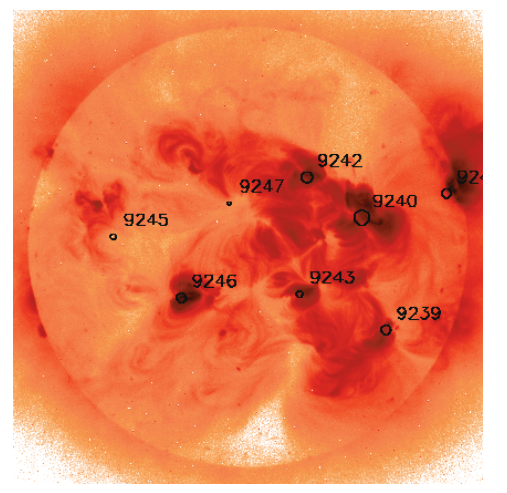

SOHO/MDI

2001-03-01 11:42

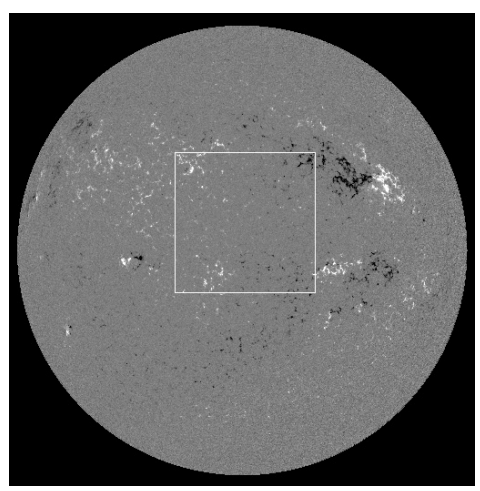

SOHO/MDI 2000-12-01 09:41

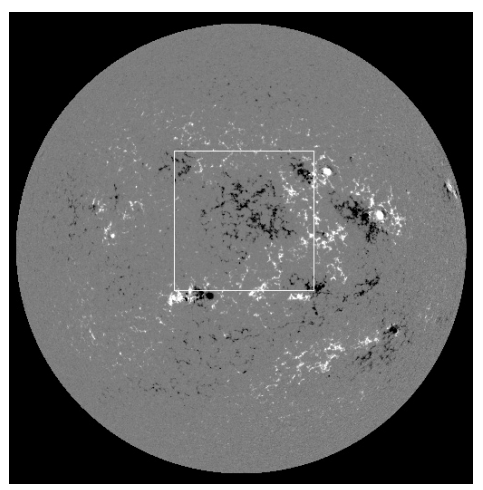

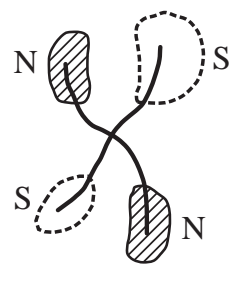

N15 W30

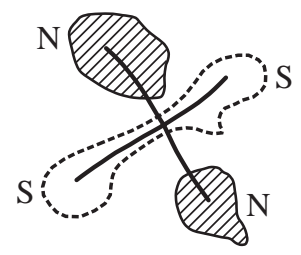

N05 W10

Figure 6: The intersecting coronal partings on 01 March 2001 at N15 W30 (top row) and on 01 December 2000 at N05 W10 (bottom row): the patterns of soft X-ray emission (left column), photospheric magnetic field (central column), and schemes of the basic coronal structures (right column). The Yohkoh SXT images in the top and bottom rows are in the inversed palettes (positive and negative). 


\subsection{Evolution of the partings}

It was already mentioned earlier that $\mathrm{CH}$ and $\mathrm{CP}$ should be considered as similar structures. Therefore, their mutual transformation is a very important issue. We can imagine, for example, the following evolution: A decaying active region becomes unipolar during its long lifetime, stretches out by the differential rotation into an approximately linear (quasi-one-dimensional) structure, and its loops extend to the neighboring AR of opposite polarity. The loops originating approximately orthogonal to the surface possess the specific thermodynamic character, because they are almost open outwards. As a result, they loose their density, temperature, and visibility. On the other hand, the denser closed loops attached to the neighboring opposite-polarity magnetic regions remain observable, i.e. a coronal parting is formed.

The evolution of a large $\mathrm{CH}$ in the course of a few revolutions of the Sun - from November 1972 to August 1973 - was traced already in one of the first studies of $\mathrm{CH}$ (Timothy et al. , 1975). When the area of this hole progressively decreased, a global coronal parting was formed in the same place by 20 June 1973 (Fig. 2b in the above-cited article). Next, in August 1973, the parting also began to decay, followed by the emergence of new active regions and BXRP. This is just the example of evolution of $\mathrm{CH}$ to $\mathrm{CP}$, as well as a subsequent disappearance of $\mathrm{CP}$.

A detailed case study of the inverse process - transformation of CP into $\mathrm{CH}$ - is still to be done.

\section{Discussion and conclusions}

A great variety of the magnetic plasma structures are realized in the solar atmosphere. They can experience, in fact, continuous transitions from one type to another.

The coronal holes and partings, considered in the present paper, possess quite similar basic features. The most important of them is presence of the open magnetic field lines. They determine the temperature regime and photometric characteristics of these structures. However, the area covered by the open field lines in CP is substantially less than in CH. So, their immediate influence on the formation of the solar wind should be substantially reduced. Nevertheless, the above-mentioned similarity between $\mathrm{CP}$ and $\mathrm{CH}$ enables them to transform into each other, thereby affecting the space weather.

It is interesting also to mention that recent numerical simulations by Chen

et al. (2015) demonstrated a surprising stability of the emissive EUV loops 
as compared to the magnetic field: while the evolving field lines successively enter and leave the narrow regions favorable for the release of energy, the bright loops remain actually in the same place. This is an additional argument for the importance of studying the large-scale arcade structures in the solar corona.

In summary, consideration of a few examples of the coronal partings performed in the present paper suggests that they possess the following basic properties:

1. A coronal parting is the elongated unipolar magnetic structure formed by two rows of arcs, which are directed to the neighboring regions of opposite polarity. Since coronal partings are formed by the magnetic loops, the structure and lifetime of $\mathrm{CP}$ are determined by the respective magnetic fields.

2. There can be global partings on the Sun, which usually connect the polar CH. Correspondingly, their characteristic size is comparable to the solar diameter (as is seen in the particular examples presented in Fig. 4); and the period of their existence is comparable to the $\mathrm{CH}$ lifetime (as follows from visual inspection of the series of additional images, not presented here).

3. The coronal partings passing through the equator do not change the sign of the magnetic field. On the other hand, there can be partings of opposite polarity that intersect each other.

4. The placement of CP and filaments are absolutely different: the filaments are localized at the top of arcs; while CP, near their footpoints.

5. Roughly speaking, the width and length of $\mathrm{CP}$ are inversely proportional to the magnetic field intensity: the longest CP are usually associated with weak background fields, stretched by the differential rotation; while short and narrow CP are associated with strong magnetic fields of the active regions (Fig. 1).

6. The system of magnetic field lines of $\mathrm{CP}$ covers a considerable area and interacts with two neighboring regions of opposite polarity. Thereby, it should play an important role in the hierarchy of the large-scale coronal magnetic fields.

7. By now, we were unable to detect any amplification of the solar wind associated with CP, which is not surprising: the area of open magnetic field lines in CP is usually quite small as compared to the coronal holes. 


\section{Acknowledgements}

We are grateful to B.V. Somov for valuable discussions and advises as well as to $S O H O$, Yohkoh, and SDO teams for the possibility to use their data.

This work was partially supported by the Russian Foundation for Basic Research, grant no. 08-02-01033a.

\section{References}

Altschuler, M.D., Trotter, D.E., \& Orrall, F.Q. Coronal holes. Sol. Phys. 26, 354-365, 1972.

Chen, F., Peter, H., Bingert, S., \& Cheung, M.C.M. Magnetic jam in the corona of the Sun. Nature Phys. 11, 492-495, 2015.

Delaboudinière, J.-P., Artzner, G.E., Brunaud, J., et al. EIT: Extremeultraviolet imaging telescope for the SOHO mission. Sol. Phys. 162, 291312, 1995.

Fuerst, E. \& Hirth, W. A coronal hole observed at $10.7 \mathrm{GHz}$ with a large single dish. Sol. Phys. 42, 157-161, 1975.

Golub, L., Krieger, A.S., Silk, J.K., Timothy, A.F., \& Vaiana, G.S. Solar X-ray bright points. Astrophys. J. 189, L93-L97, 1974.

Krieger, A.S., Timothy, A.F., \& Roelof, E.C. A coronal hole and its identification as the source of a high velocity solar wind stream. Sol. Phys. 29, 505-525, 1973.

Martres, M.J., Michard, R., \& Soru-Iscovici, I. Étude morphologique de la structure magnétique des régions actives en relation avec les phénomènes chromosphériques et les éruptions solaires: II. Localisation des plages brillantes, filaments et éruptions. Ann. Astrophys. 29, 249-253, 1966.

Molodenskii, M.M. \& Starkova, L.I. Solar structures related to coronal holes. Astron. Rep. 51, 1036-1041, 2007.

Munro, R.H. \& Withbroe, G.L. Properties of a coronal "hole" derived from extreme-ultraviolet observations. Astrophys. J. 176, 511-520, 1972.

Neupert, W.M. \& Pizzo, V. Solar coronal holes as sources of recurrent geomagnetic disturbances. J. Geophys. Res. 79, 3701-3709, 1974. 
Nikulin I.F. The structures of solar corona akin to the coronal holes. In Proc. Conf. "Topical Problems in the Physics of Solar and Stellar Activity", ed. V.V. Zaitsev, IPF RAN (N. Novgorod, Russia), v. 1, p. 22-25, 2003 (in Russian).

Nikulin I.F. Coronal partings: Observational features of magnetic separatrices. Bull. Rus. Acad. Sci.: Physics 70, 87-90, 2006.

Nolte, J.T., Krieger, A.S., Timothy, A.F., Vaiana, G.S., \& Zombeck, M.V. An atlas of coronal hole boundary positions May 28 to November 21, 1973. Sol. Phys. 46, 291-301, 1976.

Pesnell, W.D., Thompson, B.J., \& Chamberlin, P.C. The solar dynamics observatory (SDO). Sol. Phys. 275, 3-15, 2012.

Scherrer, P.H., Bogart, R.S., Bush, R.I., et al. The solar oscillations investigation - Michelson Doppler imager. Sol. Phys. 162, 129-188, 1995.

Somov, B.V. Plasma Astrophysics, Part II: Reconnection and Flares, Springer (NY), 2013.

Timothy, A.F., Krieger, A.S., \& Vaiana, G.S. The structure and evolution of coronal holes. Sol. Phys. 42, 135-156, 1975.

Tsuneta, S., Acton, L., Bruner, M., et al. The soft X-ray telescope for the SOLAR-A mission. Sol. Phys. 136, 37-67, 1991. 